\section{Metabolic Syndrome, Gut Microbiome and Dietary Bioactive Peptides, an Unexplored Triad}

\author{
Rohita Sinha ${ }^{1{ }^{*}}$ Jennifer Clarke, ${ }^{1,2}$ Jean-Jack Riethoven ${ }^{3,4}$
}

CrossMark

\section{ABSTRACT}

The gut microbiome is a complex, biochemically rich and essential component of the human metabolic system. It has been our understanding for very long that the gut microbes are primarily there to digest the undigested food (mainly fibers), get nourishment, and in return release metabolites helping host cells — short-chain fatty acids produced by gut microbes are a great source of energy for the colonocytes. It is only in the last decade, with advancements of DNA

sequencing platforms, that we are lettered about the association between the gut microbial composition and metabolic disorders such as obesity, dysglycemia, dyslipidemia, and cardiovascular diseases. This creates a momentum to understand the factors shaping the composition of the gut-microbiome, nature of dysbiosis (perturbation of gut microbial composition) associated with human health and ways to modulate the gut microbiome to achieve the desired health benefits
*Correspondence to: Rohita Sinha, Ph.D., Research Assistant Professor, University of Nebraska-Lincoln, Department of Food Science \& Technology rsinha2@unl.edu

Cite This Article: Sinha, R., Clarke, J., Riethoven, J. 2018. Metabolic Syndrome, Gut Microbiome and Dietary Bioactive Peptides, an Unexplored Triad. Diabesity 4(1): 1-4. DOI: 10.15562/ diabesity.2018.45

Keyword: gut microbiome, obesity, bioactive peptides

\section{GUT MICROBIOME AND METABOLIC SYNDROME}

The gut microbiome is a complex, biochemically rich and essential component of the human metabolic system. ${ }^{1}$ It has been our understanding for very long that the gut microbes are primarily there to digest the undigested food (mainly fibers), get nourishment, and in return release metabolites helping host cells short-chain fatty acids produced by gut microbes are a great source of energy for the colonocytes. ${ }^{2,3,4}$ It is only in the last decade, with advancements of DNA sequencing platforms, that we are lettered about the association between the gut microbial composition and metabolic disorders such as obesity, dysglycemia, dyslipidemia, and cardiovascular diseases. ${ }^{5,6,7}$ This creates a momentum to understand the factors shaping the composition of the gut-microbiome, nature of dysbiosis (perturbation of gut microbial composition) associated with human health and ways to modulate the gut microbiome to achieve the desired health benefits. ${ }^{8,9,10}$

\section{DIET, A PREDOMINANT FACTOR SHAPING THE GUT MICROBIOME}

Since microbes colonize gut mainly for nutrients, diet was hypothesized to be one of the predominant factors shaping the gut microbial composition. In last few years, this hypothesis was tested by multiple individual groups and reported a direct association between specific diet-types and gut microbial composition. ${ }^{1,11,12,13,14}$ Jansson/Tanja et al ${ }^{15}$ have found that a diet rich in resistant starch (RS) modulates the Firmicutes and Bacteroidetes ratio. A comparison of animal and plant based diet has shown that even a short term perturbation of diet may change the composition of the gut microbiome, and animalbased diet boosts the abundance of bile-tolerant microbes and decreases Firmicutes which uses polysaccharides from plant sources. ${ }^{16}$ Similar observation of quick changes in microbial composition has been found through the Western-diet (high Fat and Sugar). ${ }^{17}$ Therefore, a dietary modulation of gut-microbiome to treat above discussed metabolic disorders is an experimental hypothesis tested at the limited scale by several independent labs. A majority of these dietary interventions were about feeding prebiotics, which is described as the food supplements for the gut microbiome. Typically fibers, indigestible to host, are used as prebiotics and have been found to have positive impact on specific members of gut-microbiome. ${ }^{18,19,20}$

\section{BIOACTIVE DIETARY MOLECULES}

Food is obviously our prime source of essential nutrients and plays a predominant role in

\footnotetext{
'Department of Food Science and Technology,

${ }^{2}$ Department of Statistics,

${ }^{3}$ School of Biological Science,

${ }^{4}$ Center for Biotechnology,

University of Nebraska, Lincoln, NE, USA.
} 
determining our health status. While existing literature has a comprehensive report about the health benefits of various food articles, our knowledge about the molecular-mechanisms exerting these benefits is still at its infancy. Advanced techniques such as Next generation sequencing, has certainly helped us untangle the complex association between diet and the gut microbiome, which consequently affects the physiological state of the human being. One of the these less explored class of bioactive molecules and molecular-mechanisms are dietary peptides and their mode of action.

\section{Bioactive peptides:}

Bioactive peptides are part of scientific exploration since the last three decades; and almost all levels of living entities such as microbes, plants, and animals either produce or have proteins that are digested to bioactive peptides by proteases. ${ }^{21}$ For example, serum albumin treated with trypsin produces peptides with antihypertensive and antioxidant activities, similarly plant sources such as wheat, rice, oat, sorghum etc. were reported to be rich sources of bioactive peptides. ${ }^{22,23}$ There are several reports of milk and other dairy products as a reservoir of bioactive peptides. ${ }^{24,25}$ The range of activities of known bioactive peptides are comprised of antihypertensive, antioxidant, anti-inflammatory and antimicrobial actions. ${ }^{26,27}$ Among these, the antimicrobial activity of bioactive peptides draws a lot of attention, as the frequency of microbial resistance to known drugs is increasing. Antimicrobial peptides (AMP) originated from the animal system are considered as the first line of defence in the area with constant exposure to pathogenic microbes. AMPs are natural product of several prokaryotes and eukaryotes and so far more than 5000 such peptides are reported in the literature. ${ }^{28,29,30}$ The most common mode of action of AMPs is dependent on their ability to interact with the negatively charged cell surface of the microbes and then insert into the hydrophobic cell membrane and disrupt it either by creating pores or making clusters. There is another class of AMPs which are absorbed (cell penetration or endocytosis) by the microbial cells and exert their activities by disrupting essential biochemical pathways. ${ }^{31,32,33,34}$

\section{Antimicrobial peptides with intracellular activities}

Beyond the common perception of antimicrobial peptides acting as microbial cell-surface disrupter, there is ample evidence of peptides with intracellular activities. ${ }^{32,33,34}$ Following are examples of antimicrobial peptides with intracellular activities:

Buforin I/II: Buforin-I is a 39 amino-acid long AMP derived from the stomach of the Bufo bufo gargarizans, an Asian toad. ${ }^{35}$ While Buforin-I has a broad spectrum antimicrobial activity, its 21 amino-acid long derivative (Buforin-II) was found to be more potent. Contrary to the majority of known AMPs, Bufroin-I/II interacts with DNA \& RNA to kill the microbes. ${ }^{36}$

Apidaecin type peptides: These peptides are generally 18-20 amino acids long and were isolated from honeybees. ${ }^{37,38}$ Predominantly uptaken through the $\mathrm{ABC}$ transporters of Gram-negative bacteria, Apidaecin peptides bind to ribosomes to disrupt bacterial protein synthesis. ${ }^{39}$

Bac7: A 60 amino-acids long peptide derived from the bovine neutrophils. Bac7 does not disrupt bacterial cell membrane, but rather travels to the cytoplasm with the help of peptide transporter protein Sbm-A. Bac7 kills bacterial cells by targeting ribosomes and hindering protein synthesis. ${ }^{40}$

Indolicidin: It is a 13 amino-acids long peptide derived from the bovine neutrophils. It interacts with double stranded DNA molecules to arrest transcription, replication and consequently kills the bacterial cell. ${ }^{41,42}$

\section{PEPTIDES AS NATURAL INHIBITORS OF PROTEIN-PROTEIN INTERACTIONS (PPIS)}

As discussed above, there are several yet to be defined biochemical interactions or 'mode of actions' of bioactive peptides - competitive inhibition of vital protein-protein interactions is one of them. Protein-protein interactions (PPI) are essential to most of the vital cellular-pathways such as transcription, translation, signal transduction, host-pathogen interactions, etc., ${ }^{43}$ and are emerging as a new class of drug targets. Thus, recently we have seen a wider effort to develop an array of PPI inhibitors (antibodies, small-ligands and peptides $)^{44,45,46}$ and extend the concept to develop microbial PPI inhibitors as a new class of antimicrobial agents.

Cellular protein-protein interaction networks are marked by the presence of hub proteins - proteins which interact with multiple individual proteins. Such proteins are vital to entire networks, and consequently are prime targets for the antimicrobial agents. Protein-protein complexes are characterized by larger interfaces, where the interactions are mediated by several short fragments (peptides). Few of these interfacial peptides called hot-segments, contribute most to the total binding energy of these complexes. The hot-segments are used as lead-molecules to develop new PPI inhibitors. ${ }^{47,48,49}$ Few of the several such successful cases are inhibitory peptides for P53 $\& M D M 2^{50}$ and XIAP \& Smac interactions $;{ }^{51}$ there 
are also instances of peptides inhibiting oligomerization of membrane proteins. ${ }^{52,53}$

\section{TRIAD OF METABOLIC DISORDERS, GUT MICROBIOME AND DIETARY PEPTIDES}

While a majority of dietary peptides are fully digested and absorbed in the small intestine, there is a significant fraction which escapes and reaches the large intestine (a major reservoir of the gut microbes) to either further be digested by microbial enzymes or engulfed by them. As detailed above, there are multiple instances of bacterial absorption of peptides leading to the inhibition of vital microbial pathways and cell death. In the light of this statement, it would be an intriguing thought to evaluate the antimicrobial activity of dietary peptides reaching the large intestine.

Given the the ability of peptides, sharing sequence identity with hot-segments at protein-protein interfaces, to competitively inhibit PPI; we propose an intriguing idea of exploring the interaction of dietary peptides (peptides originating from digestion of dietary proteins in the human gastrointestinal tract) with gut microbes and test if such peptides can be subcategorized as new class of antimicrobial peptides. There are several unfathomed aspects of these putative interactions, such as the optimum length and composition of peptides for microbial absorption or surface binding, minimum inhibitory concentration of peptides, dietary proteins which are a rich source of such bioactive peptides, and finally fast and robust computational protocols to predict bioactive peptides and evaluate their ability to inhibit protein-protein interactions. We hope that with our increasing understanding of the composition of the gut microbiome and its association with human health, there would be significant interest among the scientific community about the interaction of gut microbes and dietary peptides. A natural extension of it would be the targeted dietary peptide based modulation of the gut microbiome to treat metabolic disorders. Authors, part of the current manuscript, are currently performing in vitro experiments to explore the absorption and microbial pathway inhibitory activities, of dietary peptides.

\section{REFERENCES}

1. E. M. M. Quigley, "Gut Bacteria in Health and Disease," Gastroenterol Hepatol (N Y), vol. 9, no. 9, pp. 560-569, Sep. 2013.

2. F. Bäckhed, R. E. Ley, J. L. Sonnenburg, D. A. Peterson, and J. I. Gordon, "Host-bacterial mutualism in the human intestine," Science, vol. 307, no. 5717, pp. 1915-1920, Mar. 2005.

3. G. den Besten, K. van Eunen, A. K. Groen, K. Venema, D.-J. Reijngoud, and B. M. Bakker, "The role of short-chain fatty acids in the interplay between diet, gut microbiota, and host energy metabolism," J. Lipid Res., vol. 54, no. 9, pp. 2325-2340, Sep. 2013.

4. D. Ríos-Covián, P. Ruas-Madiedo, A. Margolles, M. Gueimonde, C. G. de los Reyes-Gavilán, and N. Salazar, "Intestinal Short Chain Fatty Acids and their Link with Diet and Human Health," Front Microbiol, vol. 7, Feb. 2016.

5. S. Devaraj, P. Hemarajata, and J. Versalovic, "The human gut microbiome and body metabolism: implications for obesity and diabetes," Clin. Chem., vol. 59, no. 4, pp. 617628, Apr. 2013

6. Z. Jie et al., "The gut microbiome in atherosclerotic cardiovascular disease," Nature Communications, vol. 8, no. 1, p. 845 , Oct. 2017.

7. C. Menni, M. A. Jackson, T. Pallister, C. J. Steves, T. D. Spector, and A. M. Valdes, "Gut microbiome diversity and high-fibre intake are related to lower long-term weight gain," Int J Obes (Lond), vol. 41, no. 7, pp. 1099-1105, Jul. 2017.

8. J. Penders et al., "Factors influencing the composition of the intestinal microbiota in early infancy," Pediatrics, vol. 118, no. 2, pp. 511-521, Aug. 2006.

9. A. C. Ericsson and C. L. Franklin, "Manipulating the Gut Microbiota: Methods and Challenges," ILAR J, vol. 56, no. 2, pp. 205-217, 2015.

10. L. Wen and A. Duffy, "Factors Influencing the Gut Microbiota, Inflammation, and Type 2 Diabetes," J. Nutr., vol. 147, no. 7, p. 1468S-1475S, 2017.

11. A. R. Moschen, V. Wieser, and H. Tilg, "Dietary Factors: Major Regulators of the Gut's Microbiota," Gut Liver, vol. 6, no. 4, pp. 411-416, Oct. 2012.

12. P. M. Miranda et al., "High salt diet exacerbates colitis in mice by decreasing Lactobacillus levels and butyrate production," Microbiome, vol. 6, p. 57, Mar. 2018.

13. C. Losasso et al., "Assessing the Influence of Vegan, Vegetarian and Omnivore Oriented Westernized Dietary Styles on Human Gut Microbiota: A Cross Sectional Study," Front Microbiol, vol. 9, p. 317, 2018.

14. S. I. Villamil, R. Huerlimann, C. Morianos, Z. Sarnyai, and G. E. Maes, "Adverse effect of early-life high-fat/highcarbohydrate ('Western') diet on bacterial community in the distal bowel of mice," Nutrition Research, vol. 50, pp. 25-36, Feb. 2018

15. T. V. Maier et al., "Impact of Dietary Resistant Starch on the Human Gut Microbiome, Metaproteome, and Metabolome," mBio, vol. 8, no. 5, pp. e01343-17, Nov. 2017.

16. L. A. David et al., "Diet rapidly and reproducibly alters the human gut microbiome," Nature, vol. 505, no. 7484 , pp. 559-563, Jan. 2014.

17. P. J. Turnbaugh, V. K. Ridaura, J. J. Faith, F. E. Rey, R. Knight, and J. I. Gordon, "The effect of diet on the human gut microbiome: a metagenomic analysis in humanized gnotobiotic mice," Sci Transl Med, vol. 1, no. 6, p. 6ra14, Nov. 2009.

18. A. Costabile, G. E. Walton, G. Tzortzis, J. Vulevic, D. Charalampopoulos, and G. R. Gibson, "Development of a bread delivery vehicle for dietary prebiotics to enhance food functionality targeted at those with metabolic syndrome," Gut Microbes, vol. 6, no. 5, pp. 300-309, 2015.

19. M. S. Desai et al., "A Dietary Fiber-Deprived Gut Microbiota Degrades the Colonic Mucus Barrier and Enhances Pathogen Susceptibility," Cell, vol. 167, no. 5, p. 1339-1353.e21, 172016.

20. D. Lin, B. Peters, R. Sinha, J. J. Goedert, R. Hayes, and J. Ahn, "Association of dietary fiber intake and gut microbiota in healthy adults.," JCO, vol. 35, no. 15_suppl, pp. 1569-1569, May 2017.

21. A. Sánchez and A. Vázquez, "Bioactive peptides: A review," Food Qual Saf, vol. 1, no. 1, pp. 29-46, Mar. 2017.

22. F. Arrutia, Á. Puente, F. A. Riera, C. Menéndez, and U. A. González, "Influence of heat pre-treatment on BSA tryptic hydrolysis and peptide release," Food Chem, vol. 202, pp. 40-48, Jul. 2016.

23. F. Arrutia, R. Rubio, and F. A. Riera, "Production and membrane fractionation of bioactive peptides from a whey protein concentrate," Journal of Food Engineering, vol. 184, pp. 1-9, Sep. 2016. 
24. S. D. Nielsen, R. L. Beverly, and D. C. Dallas, "Milk Proteins Are Predigested Within the Human Mammary Gland," J Mammary Gland Biol Neoplasia, vol. 22, no. 4, pp. 251-261, Dec. 2017.

25. M. G. Basilicata et al., "Peptidome profiles and bioactivity elucidation of buffalo-milk dairy products after gastrointestinal digestion," Food Res. Int., vol. 105, pp. 1003-1010, Mar. 2018.

26. E. B.-M. Daliri, D. H. Oh, and B. H. Lee, "Bioactive Peptides," Foods, vol. 6, no. 5, Apr. 2017.

27. F. Bamdad, S. H. Shin, J.-W. Suh, C. Nimalaratne, and H. Sunwoo, "Anti-Inflammatory and Antioxidant Properties of Casein Hydrolysate Produced Using High Hydrostatic Pressure Combined with Proteolytic Enzymes," Molecules, vol. 22, no. 4, Apr. 2017.

28. A. A. Bahar and D. Ren, "Antimicrobial Peptides," Pharmaceuticals (Basel), vol. 6, no. 12, pp. 1543-1575, Nov. 2013.

29. T. Rončević et al., "Antibacterial activity affected by the conformational flexibility in glycine-lysine-based $\alpha$-helical antimicrobial peptides," J. Med. Chem., Mar. 2018.

30. J. Chen et al., "Aggregation-Induced Emission Probe for Study of the Bactericidal Mechanism of Antimicrobial Peptides," ACS Appl Mater Interfaces, Mar. 2018.

31. B. Bechinger and S.-U. Gorr, "Antimicrobial Peptides: Mechanisms of Action and Resistance," J. Dent. Res., vol. 96, no. 3, pp. 254-260, 2017.

32. S. T. Henriques, M. N. Melo, and M. A. R. B. Castanho, "Cell-penetrating peptides and antimicrobial peptides: how different are they?," Biochem J, vol. 399, no. Pt 1, pp. 1-7, Oct. 2006.

33. J. G. Rodriguez Plaza et al., "Cell penetrating peptides and cationic antibacterial peptides: two sides of the same coin," J. Biol. Chem., vol. 289, no. 21, pp. 14448-14457, May 2014.

34. J. S. Bahnsen, H. Franzyk, E. J. Sayers, A. T. Jones, and H. M. Nielsen, "Cell-penetrating antimicrobial peptides prospectives for targeting intracellular infections," Pharm. Res., vol. 32, no. 5, pp. 1546-1556, May 2015.

35. C. B. Park, M. S. Kim, and S. C. Kim, "A novel antimicrobial peptide from Bufo bufo gargarizans," Biochem. Biophys. Res. Commun., vol. 218, no. 1, pp. 408-413, Jan. 1996.

36. C. B. Park, H. S. Kim, and S. C. Kim, "Mechanism of action of the antimicrobial peptide buforin II: buforin II kills microorganisms by penetrating the cell membrane and inhibiting cellular functions," Biochem. Biophys. Res. Commun., vol. 244, no. 1, pp. 253-257, Mar. 1998.

37. W.-F. Li, G.-X. Ma, and X.-X. Zhou, "Apidaecin-type peptides: biodiversity, structure-function relationships and mode of action," Peptides, vol. 27, no. 9, pp. 2350-2359, Sep. 2006.

38. P. Casteels, C. Ampe, F. Jacobs, M. Vaeck, and P. Tempst, "Apidaecins: antibacterial peptides from honeybees," EMBO J., vol. 8, no. 8, pp. 2387-2391, Aug. 1989.

39. T. Florin et al., "An antimicrobial peptide that inhibits translation by trapping release factors on the ribosome," Nat. Struct. Mol. Biol., vol. 24, no. 9, pp. 752-757, Sep. 2017.
40. M. Mardirossian et al., "The host antimicrobial peptide Bac71-35 binds to bacterial ribosomal proteins and inhibits protein synthesis," Chem. Biol., vol. 21, no. 12, pp. 16391647, Dec. 2014.

41. M. E. Selsted, M. J. Novotny, W. L. Morris, Y. Q. Tang, W. Smith, and J. S. Cullor, "Indolicidin, a novel bactericidal tridecapeptide amide from neutrophils," J. Biol. Chem., vol. 267, no. 7, pp. 4292-4295, Mar. 1992.

42. T. J. Falla, D. N. Karunaratne, and R. E. Hancock, "Mode of action of the antimicrobial peptide indolicidin," J. Biol. Chem., vol. 271, no. 32, pp. 19298-19303, Aug. 1996.

43. G. Zinzalla and D. E. Thurston, "Targeting protein-protein interactions for therapeutic intervention: a challenge for the future," Future Med Chem, vol. 1, no. 1, pp. 65-93, Apr. 2009.

44. K. Zobel et al., "Design, synthesis, and biological activity of a potent Smac mimetic that sensitizes cancer cells to apoptosis by antagonizing IAPs," ACS Chem. Biol., vol. 1, no. 8, pp. 525-533, Sep. 2006.

45. C. D. Thanos, W. L. DeLano, and J. A. Wells, "Hot-spot mimicry of a cytokine receptor by a small molecule," Proc. Natl. Acad. Sci. U.S.A., vol. 103, no. 42, pp. 15422-15427, Oct. 2006.

46. N. C. Wrighton et al., "Small peptides as potent mimetics of the protein hormone erythropoietin," Science, vol. 273, no. 5274, pp. 458-464, Jul. 1996.

47. N. London, B. Raveh, D. Movshovitz-Attias, and O. Schueler-Furman, "Can self-inhibitory peptides be derived from the interfaces of globular protein-protein interactions?", Proteins, vol. 78, no. 15, pp. 3140-3149, Nov. 2010.

48. Y. Xu, N. A. B. D. Rahman, R. Othman, P. Hu, and M. Huang, "Computational identification of self-inhibitory peptides from envelope proteins," Proteins, vol. 80, no. 9, pp. 2154-2168, Aug. 2012.

49. L. O. Sillerud and R. S. Larson, "Design and structure of peptide and peptidomimetic antagonists of protein-protein interaction," Curr. Protein Pept. Sci., vol. 6, no. 2, pp. 151169, Apr. 2005.

50. K. Sakurai, H. S. Chung, and D. Kahne, "Use of a retroinverso p53 peptide as an inhibitor of MDM2," J. Am. Chem. Soc., vol. 126, no. 50, pp. 16288-16289, Dec. 2004.

51. Z. Liu et al., "Structural basis for binding of Smac/DIABLO to the XIAP BIR3 domain," Nature, vol. 408, no. 6815, pp. 1004-1008, Dec. 2000

52. H. Yin et al., "Computational design of peptides that target transmembrane helices," Science, vol. 315, no. 5820, pp. 1817-1822, Mar. 2007.

53. S. J. Shandler et al., "Computational design of a $\beta$-peptide that targets transmembrane helices," J. Am. Chem. Soc., vol. 133, no. 32, pp. 12378-12381, Aug. 2011.

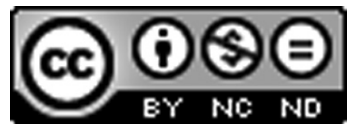

This work is licensed under a Creative Commons Attribution-Non Commercial-No Derivatives 4.0 International License. To view a copy of this license, visit http://creativecommons.org/licenses/by-nc-nd/4.0/ 